Pacific Journal of Mathematics

STANDARD ALGEBRAS 


\title{
STANDARD ALGEBRAS
}

\author{
R. D. SCHAFER
}

In $1948 \mathrm{~A}$. A. Albert defined a standard algebra $\mathfrak{A}$ by the identities $(x, y, z)+(z, x, y)-(x, z, y)=0$ and

$$
(x, y, w z)+(w, y, x z)+(z, y, w x)=0 .
$$

Standard algebras include all associative algebras and commutative Jordan algebras. The radical $\mathfrak{N}$ of any finite-dimensional standard algebra $\mathfrak{A}$ is its maximal nilpotent ideal. It is known that any semisimple standard algebra is a direct sum of simple ideals, and that any simple standard algebra is either associative or a commutative Jordan algebra.

In this paper we study Peirce decompositions and derivations of standard algebras. We prove the Wedderburn principal theorem for standard algebras of characteristic $\neq 2$ (announced in 1950 by A. J. Penico for characteristic 0$)$ : if $\mathscr{U} / \mathfrak{N}$ is separable, then $\mathfrak{U}=\mathfrak{S}+\mathfrak{N}$ where $\mathfrak{S}$ is a subalgebra of $\mathfrak{A}, \mathfrak{S} \cong \mathfrak{U} / \mathfrak{R}$. For standard algebras of characteristic 0 we prove analogues of the Malcev-Harish-Chandra theorem and the first Whitehead lemma, and we determine when the derivation algebra of $\mathfrak{U}$ is semisimple.

Let $\mathfrak{A}$ be a nonassociative algebra over a field $F$ of characteristic $\neq 2$. In [2] Albert called $\mathfrak{U}$ a standard algebra in case the identities

$$
(x, y, z)+(z, x, y)-(x, z, y)=0
$$

and

$$
(x, y, w z)+(w, y, x z)+(z, y, w x)=0
$$

are satisfied, where $(x, y, z)$ denotes the associator

$$
(x, y, z)=(x y) z-x(y z) \text {. }
$$

Clearly every associative algebra is a standard algebra. In every nonassociative algebra one has the identity

$$
(x, y, z)+(z, x, y)-(x, z, y)=[x y, z]-[x, z] y-x[y, z]
$$

where $[x, y]$ denotes the commutator $[x, y]=x y-y x$. Hence (1) is equivalent to

$$
[x y, z]=[x, z] y+x[y, z],
$$

so that every commutative algebra satisfies (1). Thus every commutative Jordan algebra of characteristic $\neq 2$ is a standard algebra.

If the characteristic is $\neq 3$, then (2) implies 


$$
\left(x, y, x^{2}\right)=0 .
$$

We shall define $\mathfrak{A}$ to be a standard algebra in case (1), (2) and (4) are satisfied. Condition (4) is redundant except for characteristic 3. Put $z=x$ in (1). Then

$$
(x, y, x)=0
$$

for all $x, y$ in $\mathfrak{A}$; that is, $\mathfrak{A}$ is flexible. Hence, as Albert proved, every standard algebra is a noncommutative Jordan algebra [18, p. 140] and is therefore power-associative. The linearized form of (5) is

$$
(x, y, z)+(z, y, x)=0 .
$$

Using flexibility, it is easy to see that, if an identity element 1 is adjoined to a standard algebra, the result is a standard algebra.

Interchange $x$ and $z$ in (2), and subtract, in order to obtain

$$
(w, y,[x, z])=0
$$

for all $w, x, y, z$ in $\mathfrak{A}$. Defining a nonassociative ring to be accessible in case (1) and (6) are satisfied, Kleinfeld proved in [8] that any simple accessible ring (of arbitrary characteristic) is either associative or commutative, implying that any simple standard ring is either associative or a commutative Jordan ring.

Albert had proved the latter result for finite-dimensional simple standard algebras in [2]. In that paper he also proved that any finite-dimensional standard nilalgebra of characteristic $\neq 2$ is nilpotent. Let the radical of a finite-dimensional standard algebra $\mathfrak{A}$ of characteristic $\neq 2$ be its maximal nilpotent $(=$ solvable $=$ nil $)$ ideal $\mathfrak{R}$, and call $\mathfrak{A}$ semisimple in case $\mathfrak{N}=0$. Then $\mathfrak{A} / \mathfrak{N}$ is semisimple. Albert showed, for standard algebras of characteristic 0 , that $\mathfrak{R}$ coincides with the radical of the commutative Jordan algebra $\mathfrak{A}^{+}$, in which multiplication is defined by

$$
x \cdot y=\frac{1}{2}(x y+y x),
$$

and that any semisimple standard algebra $\mathfrak{A}$ is the direct sum

$$
\mathfrak{U}=\mathfrak{S}_{1} \oplus \mathfrak{S}_{2} \oplus \cdots \oplus \mathfrak{S}_{r}
$$

of simple ideals $\mathfrak{S}_{i}$.

The restriction to characteristic 0 is not necessary, as may be seen as follows. A nodal algebra is a power-associative algebra $\mathfrak{A}$ with 1 over $F$ such that every element of $\mathfrak{U}$ is of the form $\alpha 1+z$ where $\alpha$ is in $F$ and $z$ is nilpotent, and $\mathfrak{A}$ is not of the form $\mathfrak{A}=$ $F 1+\mathfrak{N}$ for $\mathfrak{N}$ a nilsubalgebra of $\mathfrak{A}$. Now every nodal algebra has a 
homomorphic image which is a simple nodal algebra [17, p. 117]. But any simple standard algebra is either associative or a commutative Jordan algebra, and therefore cannot be a nodal algebra. Since $\mathfrak{A}_{K}$ is a standard algebra for any scalar extension $K$ of the base field, $\mathfrak{A}_{K}$ is without nodal subalgebras, and Theorems 3 and 4 of [17] imply that, for characteristic $\neq 2, \mathfrak{R}$ coincides with the radical of $\mathfrak{A}^{+}$and that any semisimple standard algebra $\mathfrak{A}$ is a direct sum (8) of simple (associative or commutative Jordan) ideals.

In [13] Penico announced the Wedderburn principal theorem for standard algebras of characteristic 0 , together with the essential portions of our Lemma 1 and Theorem 3 which appear in $\S 4$. However, his proofs of these results, which are generalizations to standard algebras of his theorems in [12] for commutative Jordan algebras, have never been published.

Albert proved in [2] several identities which will be useful in this paper. Using flexibility, we may rewrite (2) as

$$
(w x, y, z)-(w, y, x z)+(w z, y, x)=0,
$$

or as

$$
(w x, y, z)+(x z, y, w)-(x, y, w z)=0 .
$$

Using (6) and flexibility, (10) may be rewritten as

$$
(x, y, z w)-(x z, y, w)+(z, y, x w)=0 .
$$

In terms of right and left multiplications of $\mathfrak{A},(9)$ and (11) are equivalent to

$$
R_{y(x z)}=R_{y} R_{x z}+R_{x}\left(R_{y z}-R_{y} R_{z}\right)+R_{z}\left(R_{y x}-R_{y} R_{x}\right)
$$

and

$$
L_{(x z) y}=L_{y} L_{x z}+L_{x}\left(L_{z y}-L_{y} L_{z}\right)+L_{z}\left(L_{x y}-L_{y} L_{x}\right)
$$

for all $x, y, z$ in any standard algebra $\mathfrak{A}$. Then (12) and (13) imply

$$
R_{x^{3}}=3 R_{x} R_{x^{2}}-2 R_{x}^{3}
$$

and

$$
L_{x^{3}}=3 L_{x} L_{x^{2}}-2 L_{x}^{3}
$$

for all $x$ in $\mathfrak{A}$.

2. The Peirce decomposition. Let $\mathfrak{A}$ be a standard algebra over $F$ of characteristic $\neq 2$. Since $\mathfrak{A}$ is a noncommutative Jordan algebra, we have a Peirce decomposition 


$$
\mathfrak{U}=\mathfrak{A}_{1}+\mathfrak{A}_{\frac{1}{2}}+\mathfrak{X}_{0}
$$

relative to any idempotent $e$ in $\mathfrak{A}$, where

$$
\mathfrak{A}_{i}=\{a \in \mathfrak{A} \mid e a=a e=i a\}, \quad i=0,1,
$$

and

$$
\mathfrak{X}_{\frac{1}{2}}=\{a \in \mathfrak{U} \mid e a+a e=a\} .
$$

It is known $\left[11\right.$, p. 118] that $\mathfrak{A}_{1}$ and $\mathfrak{A}_{0}$ are orthogonal subalgebras of $\mathfrak{A}$, and that

$$
\begin{array}{ll}
\mathfrak{U}_{1} \mathfrak{A}_{\frac{1}{2}} \subseteq \mathfrak{A}_{\frac{1}{2}}, & \mathfrak{A}_{\frac{1}{2}} \mathfrak{A}_{1} \subseteq \mathfrak{U}_{\frac{1}{2}}, \\
\mathfrak{U}_{0} \mathfrak{A}_{\frac{1}{2}} \subseteq \mathfrak{A}_{\frac{1}{2}}, & \mathfrak{A}_{\frac{1}{2}} \mathfrak{A}_{0} \subseteq \mathfrak{A}_{\frac{1}{2}}
\end{array}
$$

Now (14) and (15) imply that

$$
\left(R_{e}-I\right)\left(R_{e}-\frac{1}{2} I\right) R_{e}=0
$$

and

$$
\left(L_{e}-I\right)\left(L_{e}-\frac{1}{2} I\right) L_{e}=0 .
$$

Hence $\mathfrak{U}_{\frac{1}{2}}$ is the vector space direct sum

$$
\mathfrak{A}_{\frac{1}{2}}=\mathfrak{A}_{10}+\mathfrak{U}_{\frac{11}{2}}+\mathfrak{U}_{01}
$$

where

$$
\mathfrak{A}_{i j}=\{a \in \mathfrak{A} \mid e a=i a, a e=j a\}, \quad\left(i+j=1 ; i, j=0, \frac{1}{2}, 1\right) .
$$

\begin{tabular}{|c|c|c|c|c|c|}
\hline & $\mathfrak{X}_{1}$ & $\mathfrak{A}_{10}$ & $\mathfrak{U}_{\frac{1}{2} \frac{1}{2}}$ & $\mathfrak{A}_{01}$ & $\mathfrak{X}_{0}$ \\
\hline $\mathfrak{A}_{1}$ & $\mathfrak{A}_{1}$ & $\mathfrak{A}_{10}$ & $\mathfrak{A}_{\frac{1}{2} \frac{1}{2}}$ & 0 & 0 \\
\hline $\mathfrak{A}_{10}$ & 0 & 0 & 0 & $\mathfrak{A}_{1}$ & $\mathfrak{U}_{10}$ \\
\hline $\mathfrak{A}_{\frac{1}{2} \frac{1}{2}}$ & $\mathfrak{U}_{\frac{1}{2} \frac{1}{2}}$ & 0 & $\mathfrak{U}_{1}+\mathfrak{U}_{0}$ & 0 & $\mathfrak{A}_{\frac{1}{2} \frac{1}{2}}$ \\
\hline $\mathfrak{A}_{01}$ & $\mathfrak{A}_{01}$ & $\mathfrak{U}_{0}$ & 0 & 0 & 0 \\
\hline $\mathfrak{U}_{0}$ & 0 & 0 & $\mathfrak{A}_{\frac{11}{2} \frac{1}{2}}$ & $\mathfrak{U}_{01}$ & $\mathfrak{X}_{0}$ \\
\hline
\end{tabular}

That is, any standard algebra $\mathfrak{A}$ has the Peirce decomposition

$$
\mathfrak{U}=\mathfrak{U}_{1}+\mathfrak{U}_{10}+\mathfrak{U}_{\frac{1}{2} \frac{1}{2}}+\mathfrak{U}_{01}+\mathfrak{U}_{0}
$$

relative to the idempotent $e$. (When $e$ is not the only idempotent involved, we write $\mathfrak{A}_{1}(e), \mathfrak{N}_{10}(e)$, etc.) We shall prove that products of these Peirce spaces are contained in the spaces indicated in the table below: 
Put $w=z=e, x=x_{k} \in \mathfrak{A}_{k}(k=0,1), y=y_{i j} \in \mathfrak{A}_{i j}\left(i, j=0, \frac{1}{2}, 1\right)$ in (10) to obtain $\left(x_{k}, y_{i j}, e\right)=0$, or $\left(x_{k} y_{i j}\right) e=j x_{k} y_{i j}$, implying

$$
\begin{aligned}
& \mathfrak{U}_{k} \mathfrak{U}_{10} \cong\left(\mathfrak{U}_{10}+\mathfrak{U}_{0}\right) \cap \mathfrak{U}_{\frac{1}{2}}=\mathfrak{U}_{10}, \\
& \mathfrak{U}_{k} \mathfrak{U}_{\frac{1}{2} \frac{1}{2}} \subseteq \mathfrak{U}_{\frac{1}{2} \frac{1}{2}}, \\
& \mathfrak{U}_{k} \mathfrak{A}_{01} \cong\left(\mathfrak{U}_{1}+\mathfrak{U}_{01}\right) \cap \mathfrak{U}_{\frac{1}{2}}=\mathfrak{U}_{01} .
\end{aligned}
$$

Putting $w=x=e, y=x_{k}, z=y_{i j}$ in (6), we have

$$
0=\left(e, x_{k},\left[e, y_{i j}\right]\right)=(i-j)\left(e, x_{k}, y_{i j}\right)=(i-j)\left(k x_{k} y_{i j}-e\left(x_{k} y_{i j}\right)\right),
$$

so that

$$
\begin{aligned}
& \mathfrak{A}_{0} \mathfrak{A}_{10} \subseteq\left(\mathfrak{A}_{01}+\mathfrak{A}_{0}\right) \cap \mathfrak{A}_{10}=0, \\
& \mathfrak{A}_{1} \mathfrak{A}_{01} \subseteq\left(\mathfrak{A}_{1}+\mathfrak{A}_{10}\right) \cap \mathfrak{A}_{01}=0 .
\end{aligned}
$$

We have verified the first and fifth rows of table (18). By flexibility we have $\left(e, y_{i j}, x_{k}\right)=0$ and $(i-j)\left(y_{i j}, x_{k}, e\right)=0$, from which the first and fifth columns of (18) may similarly be verified.

Put $z=e, x=x_{i j} \in \mathfrak{A}_{i j}, y=y_{k l} \in \mathfrak{A}_{k l}\left(i, j, k, l=0, \frac{1}{2}, 1\right)$ in (3) to obtain $\left[x_{i j} y_{k l}, e\right]=(j-i+l-k) x_{i j} y_{k l}$. Writing

$$
x_{i j} y_{k l}=a_{1}+a_{10}+a_{\frac{1}{2} \frac{1}{2}}+a_{01}+a_{0} \quad\left(a_{p} \in \mathfrak{A}_{p}, a_{p q} \in \mathfrak{A}_{p q}\right),
$$

we have $\left[x_{i j} y_{k l}, e\right]=-a_{10}+a_{01}$, so that

$$
\mathfrak{A}_{i j} \mathfrak{A}_{k l} \cong \mathfrak{A}_{1}+\mathfrak{A}_{\frac{1}{2} \frac{1}{2}}+\mathfrak{A}_{0} \quad \text { if } j-i+l-k=0,
$$

while

$$
\mathfrak{A}_{i j} \mathfrak{A}_{k l} \cong \mathfrak{A}_{10}+\mathfrak{A}_{01} \quad \text { if } j-i+l-k \neq 0 .
$$

Also $(j-i+l-k+1) a_{10}=(j-i+l-k-1) a_{01}=0$, implying

$$
\mathfrak{A}_{10} \mathfrak{A}_{10}=\mathfrak{A}_{01} \mathfrak{A}_{01}=0 \text {. }
$$

Put $w=x_{i j}, x=y_{k l}, y=z=e$ in (9) to obtain

$$
\left(\left(x_{i j} y_{k l}\right) e\right) e-\left(x_{i j} y_{k l}\right) e+(j-k)(j-l) x_{i j} y_{k l}=0
$$

or

$$
-\frac{1}{4} a_{\frac{1}{2} \frac{1}{2}}+(j-k)(j-l)\left(a_{1}+a_{10}+a_{\frac{1}{2} \frac{1}{2}}+a_{01}+a_{0}\right)=0 .
$$

Hence

$$
\mathfrak{A}_{i j} \mathfrak{A}_{k l} \subseteq \mathfrak{A}_{\frac{1}{2} \frac{1}{2}} \quad \text { if } \quad(j-k)(j-l) \neq 0,
$$

while

$$
\mathfrak{A}_{i j} \mathfrak{A}_{k l} \cong \mathfrak{A}_{1}+\mathfrak{A}_{10}+\mathfrak{A}_{01}+\mathfrak{A}_{0} \quad \text { if } \quad(j-k)(j-l)=0 .
$$


Then

$$
\begin{aligned}
& \mathfrak{U}_{10} \mathfrak{U}_{\frac{11}{2} \frac{1}{2}} \subseteq\left(\mathfrak{U}_{10}+\mathfrak{U}_{01}\right) \cap \mathfrak{U}_{\frac{1}{2} \frac{1}{2}}=0, \\
& \mathfrak{A}_{10} \mathfrak{A}_{01} \subseteq\left(\mathfrak{A}_{1}+\mathfrak{A}_{\frac{1}{2} \frac{1}{2}}+\mathfrak{A}_{0}\right) \cap\left(\mathfrak{A}_{1}+\mathfrak{A}_{10}+\mathfrak{A}_{01}+\mathfrak{A}_{0}\right)=\mathfrak{A}_{1}+\mathfrak{A}_{0}, \\
& \mathfrak{U}_{\frac{1}{2} \frac{1}{2}} \mathfrak{A}_{10} \subseteq\left(\mathfrak{U}_{10}+\mathfrak{U}_{01}\right) \cap \mathfrak{U}_{\frac{1}{2} \frac{1}{2}}=0 \text {, } \\
& \mathfrak{U}_{\frac{1}{2} \frac{1}{2}} \mathfrak{U}_{\frac{1}{2} \frac{1}{2}} \subseteq\left(\mathfrak{A}_{1}+\mathfrak{U}_{\frac{11}{2} \frac{1}{2}}+\mathfrak{U}_{0}\right) \cap\left(\mathfrak{U}_{1}+\mathfrak{A}_{10}+\mathfrak{A}_{01}+\mathfrak{U}_{0}\right)=\mathfrak{A}_{1}+\mathfrak{A}_{0} \text {, } \\
& \mathfrak{U}_{\frac{11}{2} \frac{1}{2}} \mathfrak{U}_{01} \subseteq\left(\mathfrak{U}_{10}+\mathfrak{A}_{01}\right) \cap \mathfrak{A}_{\frac{1}{2} \frac{1}{2}}=0 \text {, } \\
& \mathfrak{A}_{01} \mathfrak{A}_{10} \subseteq\left(\mathfrak{U}_{1}+\mathfrak{U}_{\frac{1}{2} \frac{1}{2}}+\mathfrak{A}_{0}\right) \cap\left(\mathfrak{U}_{1}+\mathfrak{U}_{10}+\mathfrak{A}_{01}+\mathfrak{A}_{0}\right)=\mathfrak{A}_{1}+\mathfrak{X}_{0} \text {, } \\
& \mathfrak{U}_{01} \mathfrak{A}_{\frac{1}{2} \frac{1}{2}} \subseteq\left(\mathfrak{A}_{10}+\mathfrak{U}_{01}\right) \cap \mathfrak{U}_{\frac{1}{2} \frac{1}{2}}=0 \text {. }
\end{aligned}
$$

It remains to be shown that

$$
\mathfrak{A}_{10} \mathfrak{A}_{01} \subseteq \mathfrak{A}_{1}+\mathfrak{A}_{0} \quad \text { implies } \quad \mathfrak{A}_{10} \mathfrak{A}_{01} \subseteq \mathfrak{A}_{1}
$$

and

$$
\mathfrak{A}_{01} \mathfrak{A}_{10} \subseteq \mathfrak{A}_{1}+\mathfrak{A}_{0} \quad \text { implies } \quad \mathfrak{A}_{01} \mathfrak{A}_{10} \subseteq \mathfrak{A}_{0} \text {. }
$$

Let $x_{i j} y_{j i}=a_{1}+a_{0}(i \neq j ; i, j=0,1)$. Putting $w=x=e, y=x_{i j}$, $z=y_{j i}$ in (6), we have $0=\left(e, x_{i j},\left[e, y_{j i}\right]\right)=(j-i)\left(i a_{1}+i a_{0}-a_{1}\right)$, or $(i-1) a_{1}+i a_{0}=0$ since $i \neq j$. Hence $a_{0}=0$ if $i=1$, while $a_{1}=0$ if $i=0$; that is, (19) and (20) hold. This completes the verification of table (18).

Relative to a set of pairwise orthogonal idempotents $e_{1}, e_{2}, \cdots, e_{t}$ in a standard algebra $\mathfrak{A}$, the Peirce decomposition (17) may be refined. However, the notation is unavoidably cumbersome. The only Peirce decomposition relative to a set of pairwise orthogonal idempotents which we shall actually use is the less complicated one which is known for noncommutative Jordan algebras [11, p.188]. We may restrict ourselves to the case where $\mathfrak{A}$ contains $1=e_{1}+e_{2}+\cdots+e_{t}$, and write $f_{i}=1-e_{i}$. Then $\mathfrak{A}$ is the vector space direct sum

$$
\mathfrak{A}=\sum_{i \leqq j} \mathfrak{A}_{i j}
$$

where

$$
\mathfrak{A}_{i i}=\mathfrak{A}_{1}\left(e_{i}\right)=\mathfrak{A}_{0}\left(f_{i}\right)
$$$$
(i=1, \cdots, t)
$$

and

$$
\mathfrak{A}_{i j}=\mathfrak{A}_{j i}=\mathfrak{A}_{\frac{1}{2}}\left(e_{i}\right) \cap \mathfrak{A}_{\frac{1}{2}}\left(e_{j}\right) \quad(i \neq j ; i, j=1, \cdots, t) .
$$

(There can be no confusion with $\mathfrak{A}_{i j}$ in (16), since here both subscripts are taken from the set $1,2, \cdots, t$, whereas in (16) at least one is not.) We have, for distinct $i, j, k, l$ the known properties:

$$
\mathfrak{U}_{i i}^{2} \subseteq \mathfrak{A}_{i i}, \quad \mathfrak{A}_{i i} \mathfrak{A}_{j j}=\mathfrak{A}_{i i} \mathfrak{A}_{j k}=\mathfrak{A}_{j k} \mathfrak{A}_{i i}=0,
$$




$$
\begin{aligned}
& \mathfrak{A}_{i i} \mathfrak{A}_{i j} \cong \mathfrak{A}_{i j}, \quad \mathfrak{U}_{i j} \mathfrak{A}_{i i} \subseteq \mathfrak{A}_{i j}, \\
& \mathfrak{A}_{i j} \mathfrak{A}_{j k} \subseteq \mathfrak{A}_{i k}, \quad \mathfrak{U}_{i j} \mathfrak{A}_{k l}=0, \\
& \mathfrak{A}_{i j}^{2} \cong \mathfrak{A}_{i i}+\mathfrak{A}_{i j}+\mathfrak{A}_{j j} .
\end{aligned}
$$

The subalgebra $\mathfrak{A}_{1}\left(e_{i}+e_{j}\right)$ is

$$
\mathfrak{A}_{1}\left(e_{i}+e_{j}\right)=\mathfrak{A}_{i i}+\mathfrak{A}_{i j}+\mathfrak{A}_{j j},
$$

and $e_{i}+e_{j}$ is its identity element.

McCrimmon's results on noncommutative Jordan algebras with a set $e_{1}, e_{2}, \cdots, e_{t}(t \geqq 3)$ of connected idempotents may be applied to standard algebras. An element $x$ in $\mathfrak{A}$ (with 1 ) is called regular [10, p. 943] in case there exists $y$ in $\mathfrak{A}$ satisfying

$$
x y=y x=1, \quad x^{2} y=y x^{2}=x .
$$

Then $e_{i}$ and $e_{j}(i \neq j)$ are said to be connected with indicator $\phi=0$ (resp. $\varphi=1 / 4$ ) in case there is an element $x$ in

$$
\left(\mathfrak{A}_{10}\left(e_{i}\right)+\mathfrak{A}_{01}\left(e_{i}\right)\right) \cap\left(\mathfrak{U}_{10}\left(e_{j}\right)+\mathfrak{A}_{01}\left(e_{j}\right)\right)
$$

(resp. $x$ in $\mathfrak{A}_{\frac{1}{2} \frac{1}{2}}\left(e_{i}\right) \cap \mathfrak{A}_{\frac{1}{2} \frac{1}{2}}\left(e_{j}\right)$ ) which is regular in the subalgebra $\mathfrak{A}_{i i}+\mathfrak{A}_{i j}+\mathfrak{A}_{j j}[11$, p. 190]. McCrimmon has proved [11, p. 191] that, in case $1=e_{1}+e_{2}+\cdots+e_{t}$ is the sum of $t \geqq 3$ connected orthogonal idempotents with indicator $\varphi=0$ (resp. $\varphi=1 / 4)$, then $\mathfrak{A}$ is associative (resp. a commutative Jordan algebra).

The radical $\mathfrak{N}_{i i}$ of $\mathfrak{N}_{i i}$ is

$$
\mathfrak{N}_{i i}=\mathfrak{A}_{i i} \cap \mathfrak{R}
$$

where $\mathfrak{N}$ is the radical of $\mathfrak{A}$. For this is true in the commutative Jordan algebra $\mathfrak{U}^{+}[7, \S 7.6$, Lemma 1]. Since we have seen in $\S 1$ that the radical of $\mathfrak{A}^{+}$is $\mathfrak{R}^{+}$, we have $\mathfrak{R}_{i i}=\mathfrak{N}_{i i}^{+}=\mathfrak{U}_{i i}^{+} \cap \mathfrak{R}^{+}=\mathfrak{A}_{i i} \cap \mathfrak{N}$.

If a standard algebra $\mathfrak{A}$ is associative, then it is well known that the Peirce space $\mathfrak{A}_{\frac{1}{2} \frac{1}{2}}$ relative to any idempotent $e$ is 0 . We remark that this readily implies that, if $\mathfrak{B}$ is an ideal in a standard algebra $\mathfrak{A}$, and if $\mathfrak{A} / \mathfrak{B}$ is associative, then $\mathfrak{A}_{\frac{1}{2} \frac{1}{2}} \subseteq \mathfrak{B}$. Similarly, if $\mathfrak{A} / \mathfrak{B}$ is a commutative Jordan algebra, then $\mathfrak{A}_{10}+\mathfrak{A}_{01} \subseteq \mathfrak{B}$.

3. Derivations. A derivation $D$ of a nonassociative algebra $\mathfrak{A}$ over $F$ is a linear operator on $\mathfrak{A}$ satisfying

$$
(x y) D=(x D) y+x(y D)
$$

for all $x, y$ in $\mathfrak{A}$. (More generally, if $\mathfrak{A}$ is a subalgebra of an algebra $\mathfrak{B}$ over $F$, a derivation of $\mathfrak{A}$ into $\mathfrak{B}$ is a linear mapping $D$ of $\mathfrak{A}$ into $\mathfrak{B}$ satisfying (24) for all $x, y$ in $\mathfrak{A}$.) The set $\mathfrak{D}(\mathfrak{A})$ of all derivations 
of a nonassociative algebra $\mathfrak{A}$ is a Lie algebra, the derivation algebra $\mathfrak{D}(\mathfrak{U})$.

In terms of right and left multications of an algebra $\mathfrak{A},(24)$ is equivalent to

$$
\left[R_{y}, D\right]=R_{y D}
$$

for all $y$ in $\mathfrak{A}$, and to

$$
\left[L_{x}, D\right]=L_{x D}
$$

for all $x$ in $\mathfrak{A}$. The Lie multiplication algebra $\mathfrak{L}(\mathfrak{H})$ of a nonassociative algebra $\mathfrak{A}$ is the enveloping Lie algebra of the right and left multiplications of $\mathfrak{A}$. A derivation $D$ of $\mathfrak{U}$ is called inner in case $D$ is in $\mathfrak{L}(\mathfrak{U})[18$, p. 21].

It is well known [18, p. 92] that, in any commutative Jordan algebra $\mathfrak{\Im}$ of characteristic $\neq 2$, the operators $\left[R_{x}, R_{y}\right]$ are derivations for all $x, y$ in $\mathfrak{\Im}$. Indeed, if $\Im$ contains 1 , then the inner derivations of $\mathfrak{\Im}$ are exactly the operators

$$
\sum_{i}\left[R_{x_{i}}, R_{y_{i}}\right] ; \quad \quad x_{i}, y_{i} \text { in } \Im .
$$

(Since in this paper we shall encounter commutative Jordan algebras which do not necessarily contain 1 , we shall simplify matters here by reserving the term inner derivation of a commutative Jordan algebra for the derivations having the form (27).)

If $\mathfrak{U}$ is a standard algebra, so that the algebra $\mathfrak{U}^{+}$defined by (7) is a commutative Jordan algebra, then the operators $\left[R_{x}^{+}, R_{y}^{+}\right]$, where

$$
R_{x}^{+}=\frac{1}{2}\left(R_{x}+L_{x}\right),
$$

are derivations of $\mathfrak{A}^{+}$. Then the inner derivations of $\mathfrak{U}^{+}$are sums of such operators $\left[R_{x}^{+}, R_{y}^{+}\right]$. According to the following theorem, they are actually derivations of $\mathfrak{A}$.

THEOREM 1. Let $\mathfrak{U}$ be a standard algebra of characteristic $\neq 2$. Then the following are derivations of $\mathfrak{A}$ :

$$
R_{d}-L_{d} \quad \text { for all } d \text { in } \mathfrak{A} \text {; }
$$

and

$$
\left[R_{x}+L_{x}, R_{y}+L_{y}\right]=R_{[x, y]}-L_{[x, y]}+4\left[L_{x}, R_{y}\right]
$$

for all $x, y$ in $\mathfrak{A}$. 
Proof. The operators $R_{d}-L_{d}$ are derivations by (3). Hence $\left[R_{x}, R_{y}-L_{y}\right]=R_{[x, y]}$ and $\left[L_{x}, R_{y}-L_{y}\right]=L_{[x, y]}$ by (25) and (26). Since $\left[R_{x}, L_{y}\right]=\left[L_{x}, R_{y}\right]$ by flexibility, we have

$$
\begin{aligned}
& {\left[R_{x}, R_{y}\right]=R_{[x, y]}+\left[L_{x}, R_{y}\right],} \\
& {\left[L_{x}, L_{y}\right]=-L_{[x, y]}+\left[L_{x}, R_{y}\right],}
\end{aligned}
$$

and the equality which is indicated in (30). Then (29) is a derivation of $\mathfrak{A}\left(\right.$ resp. $\left.\mathfrak{A}^{+}\right)$if and only if $(30)$ is a derivation of $\mathfrak{A}$ (resp. $\mathfrak{A}^{+}$). But we already know that (30) is a derivation of $A^{+}$. Hence

$$
\left[R_{z}+L_{z},\left[L_{x}, R_{y}\right]\right]=R_{(x, z, y)}+L_{(x, z, y)}
$$

for all $x, y, z$ in $\mathfrak{A}$. Now (6), (1) and flexibility imply that

$$
(x,[w, z], y)=0
$$

for all $x, y, z, w$ in $\mathfrak{A}$, or

$$
R_{z}\left[L_{x}, R_{y}\right]=L_{z}\left[L_{x}, R_{y}\right] .
$$

Since $R_{w}-L_{w}$ is a derivation of $\mathfrak{A}$, we have

$$
[(x, z, y), w]=([x, w], z, y)+(x,[z, w], y)+(x, z,[y, w]) .
$$

But each term on the right side of this equation is 0 , so

$$
[(x, z, y), w]=0
$$

for all $x, y, z, w$ in $\mathfrak{X}[8, \mathrm{p} .336]$, or

$$
L_{(x, z, y)}=R_{(x, z, y)} \cdot
$$

Interchanging $w$ and $z$ in (35), we have $[(x, w, y), z]=0$, so that

$$
\left[L_{x}, R_{y}\right] R_{z}=\left[L_{x}, R_{y}\right] L_{z} \text {. }
$$

Then (33), (34), (36) and (37) imply $\left[R_{z},\left[L_{x}, R_{y}\right]\right]=R_{(x, z, y)}=R_{z\left[L_{x}, R_{y}\right]}$, so that $\left[L_{x}, R_{y}\right]$ is a derivation of $\mathfrak{A}$.

CoRollary. Let $\mathfrak{A}$ be a standard algebra over $F$ of characteristic $\neq 2$. Then any inner derivation of the commutative Jordan algebra $\mathfrak{A}^{+}$is a derivation of $\mathfrak{A}^{\text {. }}$

THEOREM 2. The Lie multiplication algebra $\mathfrak{2}(\mathfrak{U})$ of any standard algebra $\mathfrak{A}$ of characteristic $\neq 2$ is

$$
\mathfrak{L}(\mathfrak{U})=R(\mathfrak{A})+L(\mathfrak{A})+[L(\mathfrak{U}), R(\mathfrak{A})],
$$

where $R(\mathfrak{A}) \quad(\operatorname{resp} . L(\mathfrak{H}))$ denotes the set of all right (resp. left) 
multiplications of $\mathfrak{A}$.

Proof. It is sufficient to verify that

$$
\mathfrak{L}=R(\mathfrak{U})+L(\mathfrak{U})+[L(\mathfrak{U}), R(\mathfrak{U})]
$$

is a Lie algebra. Now (31) and (32) imply that $\left[R_{x}, R_{y}\right]$ and $\left[L_{x}, L_{y}\right]$ are in $\mathbb{Z}$ for all $x, y$ in $\mathfrak{A}$. Also Theorem 1 implies that

$$
\left[R_{z},\left[L_{x}, R_{y}\right]\right]=R_{(x, z, y)}
$$

and

$$
\left[L_{z},\left[L_{x}, R_{y}\right]\right]=L_{(x, z, y)}
$$

are in $\mathfrak{Q}$ for all $x, y, z$ in $\mathfrak{A}$ by (25) and (26). Finally,

$$
\begin{aligned}
{\left[\left[L_{a}, R_{b}\right],\left[L_{x}, R_{y}\right]\right] } & =-\left[\left[R_{b},\left[L_{x}, R_{y}\right]\right], L_{a}\right]-\left[\left[\left[L_{x}, R_{y}\right], L_{a}\right], R_{b}\right] \\
& =\left[L_{a}, R_{(x, b, y)}\right]+\left[L_{(x, a, y)}, R_{b}\right]
\end{aligned}
$$

is in $\mathbb{Q}$ for all $a, b, x, y$ in $\mathfrak{A}$ by the Jacobi identity.

CoRollary. Let $\mathfrak{A}$ be a standard algebra with 1 over $F$ of characteristic $\neq 2$. The inner derivations of $\mathfrak{A}$ are the operators

$$
R_{d}-L_{d}+\sum_{i}\left[L_{x_{i}}, R_{y_{i}}\right], \quad d, x_{i}, y_{i} \text { in } \mathfrak{A} \text {. }
$$

Proof. Let $D=R_{d}+L_{f}+\sum_{i}\left[L_{x_{i}}, R_{y_{i}}\right]$ in $\mathfrak{R}(\mathfrak{H})$ be a derivation of भ. Then $0=1 D=d+f$, implying $D$ has the form (38). But any such $D$ is a derivation by Theorem 1 .

4. The Wedderburn principal theorem. Our chief result in this section is a generalization of both the Wedderburn principal theorem for associative algebras [1, p. 47] and its analogue for commutative Jordan algebras [12; 7, Chapter VII]. We shall use both of these theorems in its proof. As we have indicated in $\S 1$, Penico has announced this result (Theorem 4) for characteristic 0, together with the essential portions of Lemma 1 and Theorem 3, in [13].

Lemma 1. Let $\mathfrak{B}$ be an ideal of a standard algebra $\mathfrak{A}$ of characteristic $\neq 2$. Then the following are also ideals of $\mathfrak{A}$ :

$$
\begin{aligned}
& \mathfrak{U} \mathfrak{B}^{2}+\mathfrak{B}^{2}=\mathfrak{B}^{2} \mathfrak{A}+\mathfrak{B}^{2}, \\
& \mathfrak{B}^{2} \mathfrak{B}+\mathfrak{B}^{2} \mathfrak{B}^{2}\left(=\mathfrak{B}^{3}\right) .
\end{aligned}
$$

Proof. Put $x=b_{1} \in \mathfrak{B}, y=b_{2} \in \mathfrak{B}, z=a \in \mathfrak{A}$ in (1) to obtain

$$
\left(b_{1} b_{2}\right) a=b_{1}\left(b_{2} a\right)-\left(a b_{1}\right) b_{2}+a\left(b_{1} b_{2}\right)+\left(b_{1} a\right) b_{2}-b_{1}\left(a b_{2}\right)
$$


in $\mathfrak{A} \mathfrak{B}^{2}+\mathfrak{B}^{2}$, implying $\mathfrak{B}^{2} \mathfrak{A} \cong \mathfrak{A B}^{2}+\mathfrak{B}^{2}$. Similarly, $\mathfrak{A} \mathfrak{B}^{2} \cong \mathfrak{B}^{2} \mathfrak{A}+\mathfrak{B}^{2}$. This establishes the equality in (39). To see that $\mathfrak{A}^{2}+\mathfrak{B}^{2}$ is a left ideal of $\mathfrak{A}$, we put $x=c \in \mathfrak{A}, y=a \in \mathfrak{A}, w=b_{1} \in \mathfrak{B}, z=b_{2} \in \mathfrak{B}$ in (2). We obtain

$$
c\left(a\left(b_{1} b_{2}\right)\right)=(c a)\left(b_{1} b_{2}\right)+\left(b_{1} a\right)\left(c b_{2}\right)-b_{1}\left(a\left(c b_{2}\right)\right)+\left(b_{2} a\right)\left(b_{1} c\right)-b_{2}\left(a\left(b_{1} c\right)\right)
$$

in $\mathfrak{A} \mathfrak{B}^{2}+\mathfrak{B}^{2}$, so that $\mathfrak{A}\left(\mathfrak{A} \mathfrak{B}^{2}+\mathfrak{B}^{2}\right) \subseteq \mathfrak{A} \mathfrak{B}^{2}+\mathfrak{B}^{2}$. Similarly, $\mathfrak{B}^{2} \mathfrak{A}+\mathfrak{B}^{2}$ is a right ideal of $\mathfrak{A}$, so that (39) is an ideal of $\mathfrak{A}$. To prove that (40) is an ideal of $\mathfrak{A}$, we first put $x=b_{1} \in \mathfrak{B}, y=b_{2} \in \mathfrak{B}, z=a \in \mathfrak{A}$ in (3) to see that

$$
\left[\mathfrak{B}^{2}, \mathfrak{U}\right] \subseteq \mathfrak{B}^{2} \text {. }
$$

Then, putting $w=b_{1} \in \mathfrak{B}, x=b_{2} \in \mathfrak{B}, y=b_{3} \in \mathfrak{B}, z=a \in \mathfrak{X}$ in (10), we have $\left(\left(b_{1} b_{2}\right) b_{3}\right) a=\left(b_{1} b_{2}\right)\left(b_{3} a\right)-\left(\left(b_{2} a\right) b_{3}\right) b_{1}+\left(b_{2} a\right)\left(b_{3} b_{1}\right)+\left(b_{2} b_{3}\right)\left(b_{1} a\right)-b_{2}\left(b_{3}\left(b_{1} a\right)\right)$ in $\mathfrak{B}^{2} \mathfrak{B}+\mathfrak{B}^{2} \mathfrak{B}^{2}$, implying $\left(\mathfrak{B}^{2} \mathfrak{B}\right) \mathfrak{A} \subseteq \mathfrak{B}^{2} \mathfrak{B}+\mathfrak{B}^{2}$. Similarly, $\mathfrak{A}\left(\mathfrak{B}^{2} \mathfrak{B}^{2}\right) \subseteq$ $\mathfrak{B}^{2} \mathfrak{B}^{2}+\mathfrak{B}^{2}$. Put $x=b \in \mathfrak{B}, y \in \mathfrak{B}^{2}, z=a \in \mathfrak{A}$ in (3) to obtain

$$
(b y) a=a(b y)+[b, a] y+b[y, a]
$$

in $\mathfrak{A}\left(\mathfrak{B}^{2}\right)+\mathfrak{B}^{2} \mathfrak{B}^{2}$ by $(41)$, implying $\left(\mathfrak{B}^{2} \mathfrak{B}^{2}\right) \mathfrak{U} \subseteq \mathfrak{B}^{2} \mathfrak{B}+\mathfrak{B}^{2} \mathfrak{B}^{2}$. Similarly, $\mathfrak{A}\left(\mathfrak{B}^{2} \mathfrak{B}\right) \subseteq \mathfrak{B}^{2} \mathfrak{B}+\mathfrak{B}_{\mathfrak{B}^{2}}$. This proves that $\mathfrak{B}^{3}$, defined as $\mathfrak{B}^{3}=\mathfrak{B}^{2} \mathfrak{B}+\mathfrak{B}^{2} \mathfrak{B}^{2}$, is an ideal of $\mathfrak{A}$.

In any nonassociative algebra $\mathfrak{A}$ the derived series

$$
\mathfrak{B}^{(0)} \supseteqq \mathfrak{B}^{(1)} \supseteqq \cdots \supseteqq \mathfrak{B}^{(k)} \supseteqq \cdots
$$

of $\mathfrak{B}$ is defined by

$$
\mathfrak{B}^{(0)}=\mathfrak{B}, \quad \mathfrak{B}^{(i+1)}=\left(\mathfrak{B}^{(i)}\right)^{2},
$$

and $\mathfrak{B}$ is called solvable in case there is some $k$ for which $\mathfrak{B}^{(k)}=0$. If $\mathfrak{B}$ is an ideal of $\mathfrak{A}$, the terms of the derived series (42) are not in general ideals of $\mathfrak{A}$. By Lemma 1 we do obtain from any ideal $\mathfrak{B}$ in a standard algebra $\mathfrak{A}$ a descending chain

$$
\mathfrak{B}^{\langle 0\rangle} \supseteqq \mathfrak{B}^{\langle 1\rangle} \supseteqq \cdots \supseteqq \mathfrak{B}^{\langle k\rangle} \supseteqq \cdots
$$

of ideals $\mathfrak{B}^{\langle i\rangle}$ of $\mathfrak{A}$ defined by

$$
\mathfrak{B}^{\langle 0\rangle}=\mathfrak{B}, \quad \mathfrak{B}^{\langle i+1\rangle}=\mathfrak{U}\left(\mathfrak{B}^{\langle i\rangle}\right)^{2}+\left(\mathfrak{B}^{\langle i\rangle}\right)^{2} .
$$

Following Jacobson's terminology for commutative Jordan algebras, we call (43) a Penico sequense and call the ideal $\mathfrak{B}$ Penico solvable in case there is some $k$ for which $\mathfrak{B}^{\langle k\rangle}=0$.

If $\mathfrak{B}$ is Penico solvable, then $\mathfrak{B}$ is solvable, since 


$$
\mathfrak{B}^{(i)} \supseteq \mathfrak{B}^{(i)}
$$$$
\text { for } i=0,1,2, \cdots \text {. }
$$

The converse is known for finite-dimensional commutative Jordan algebras [12; 7, Chapter V]. We model our proof for standard algebras on an unpublished concise proof by K. McCrimmon for the commutative Jordan case.

The multiplication algebra $\mathfrak{M}(\mathfrak{A})$ of any nonassociative algebra $\mathfrak{A}$ is the enveloping associative algebra of the right and left multiplications of $\mathfrak{A}$. If $\mathfrak{B}, \mathfrak{C}$ are ideals of $\mathfrak{A}$, then

$$
[\mathfrak{C}: \mathfrak{B}]=\{T \in \mathfrak{M}(\mathfrak{A}) \mid \mathfrak{B} T \subseteq \mathfrak{\subseteq}\}
$$

is an ideal of $\mathfrak{M}(\mathfrak{U})$. Lemma 1 implies that, if $\mathfrak{B}$ is an ideal of a standard algebra $\mathfrak{A}$, then

$$
\mathfrak{Q}=\left[\mathfrak{B}^{3}: \mathfrak{B}\right]
$$

is an ideal of $\mathfrak{M}(\mathfrak{A})$. Also

$$
\mathfrak{B D} \subseteq B^{3} .
$$

Theorem 3. Any solvable ideal $\mathfrak{B}$ in a finite-dimensional standard algebra $\mathfrak{A}$ over $F$ of characteristic $\neq 2$ is Penico solvable.

Proof. It is sufficient to prove that, if $\mathfrak{B}$ is any ideal of finite codimension in a (possibly infinite-dimensional) standard algebra $\mathfrak{A}$, then

$$
\mathfrak{B}^{\langle n+1\rangle} \cong \mathfrak{B}^{3} \quad \text { if } 2 n-1>\operatorname{dim} \mathfrak{A} / \mathfrak{B} \text {. }
$$

For (47) and the assumed finite-dimensionality of $\mathfrak{A}$ insure that there is an integer $t$ such that $\mathfrak{C}^{\langle t\rangle} \subseteq \mathfrak{\complement}^{3} \subseteq \mathfrak{C}^{2}=\mathfrak{C}^{(1)}$ for every ideal $\mathfrak{c}$ of $\mathfrak{A}$. Then $\mathfrak{B}^{\langle k t\rangle} \subseteq \mathfrak{B}^{(k)}$ for any ideal $\mathfrak{B}$, as may be seen by induction:

$$
\mathfrak{B}^{\langle(k+1) t\rangle}=\left(\mathfrak{B}^{\langle k t\rangle}\right)^{\langle t\rangle} \subseteq\left(\mathfrak{B}^{\langle k t\rangle}\right)^{(1)} \cong\left(\mathfrak{B}^{(k)}\right)^{(1)}=\mathfrak{B}^{(k+1)} \text {. }
$$

If $\mathfrak{B}$ is solvable, then $\mathfrak{B}^{\langle k t\rangle} \subseteq \mathfrak{B}^{(k)}=0$ for some $k$, and $\mathfrak{B}$ is Penico solvable.

In order to prove (47), we adjoin 1 to $\mathfrak{A}$ to obtain $\mathfrak{A}_{1}=F 1+\mathfrak{A}$, so that $\mathfrak{B}^{\langle i+1\rangle}=\mathfrak{A}_{1}\left(\mathfrak{B}^{\langle i\rangle}\right)^{2}$ in (44). We shall show first that, for any $n$,

$$
\mathfrak{B}^{\langle n+1\rangle} \subseteq \mathfrak{B}\left(L\left(\mathfrak{B}, \mathfrak{A}_{1}\right) L\left(\mathfrak{A}_{1}\right)\right)^{2 n}+\mathfrak{B}^{3},
$$

where $L\left(\mathfrak{B}, \mathfrak{A}_{1}\right)$ denotes the set of all left multiplications of $\mathfrak{A}_{1}$ corresponding to elements of $\mathfrak{B}$. For $\mathfrak{Q}$ in (45) we have

$$
L_{b_{1} b_{2}} \in \mathfrak{Q}, \quad L_{b_{1}} L_{b_{2}} \in \mathfrak{Q}
$$

for all $b_{i}$ in $\mathfrak{B}$. Put $x=b_{1} \in \mathfrak{B}, z=b_{2} \in \mathfrak{B}, y=a \in \mathfrak{A}_{1}$ in (13) to obtain 


$$
L_{\left(b_{1} b_{2}\right) a} \equiv-L_{b_{2}} L_{a} L_{b_{1}}-L_{b_{1}} L_{a} L_{b_{2}} \quad(\bmod \mathfrak{\Omega})
$$

by (49), implying

$$
L\left(\mathfrak{B}^{2} \mathfrak{A}_{1}, \mathfrak{A}_{1}\right) \subseteq L\left(\mathfrak{B}, \mathfrak{A}_{1}\right) L\left(\mathfrak{A}_{1}\right) L\left(\mathfrak{B}, \mathfrak{A}_{1}\right)+\mathfrak{Q} .
$$

We prove (48) by induction on $n$. The case $n=0$ is clear. Assuming (48), we have

$$
\begin{aligned}
\mathfrak{B}^{\langle n+2\rangle} & =\mathfrak{A}_{1}\left(\mathfrak{B}^{\langle n+1\rangle}\right)^{2} \\
& \cong \mathfrak{A}_{1}\left(\mathfrak{B}^{\langle 1\rangle} \mathfrak{B}^{\langle n+1\rangle}\right) \\
& =\mathfrak{B}^{\langle n+1\rangle} L\left(\mathfrak{B}^{\langle 1\rangle}, \mathfrak{N}_{1}\right) L\left(\mathfrak{A}_{1}\right) \\
& \subseteq \mathfrak{B}^{\langle n+1\rangle} L\left(\mathfrak{B}, \mathfrak{A}_{1}\right) L\left(\mathfrak{A}_{1}\right) L\left(\mathfrak{B}, \mathfrak{A}_{1}\right) L\left(\mathfrak{U}_{1}\right)+\mathfrak{B}^{3} \\
& \subseteq \mathfrak{B}\left(L\left(\mathfrak{B}, \mathfrak{A}_{1}\right) L\left(\mathfrak{A}_{1}\right)\right)^{2(n+1)}+\mathfrak{B}^{3}
\end{aligned}
$$

by (50) and (46), as desired. Put $x=a_{1} \in \mathfrak{A}_{1}, \quad z=a_{2} \in \mathfrak{A}_{1}, \quad y=b_{2} \in \mathfrak{B}$ in (13) to obtain

$$
L_{\left(a_{1} a_{2}\right) b_{2}}=L_{b_{2}} L_{a_{1} a_{2}}+L_{a_{2}}\left(L_{a_{1} b_{2}}-L_{b_{2}} L_{a_{1}}\right)+L_{a_{1}}\left(L_{a_{2} b_{2}}-L_{b_{2}} L_{a_{2}}\right) .
$$

Multiply (51) on the left by $L_{b_{1}}$ and on the right by $L_{b_{3}}$, for $b_{i} \in \mathfrak{B}$, to obtain

$$
L_{b_{1}}\left(L_{a_{2}} L_{b_{2}} L_{a_{1}}+L_{a_{1}} L_{b_{2}} L_{a_{2}}\right) L_{b_{3}} \in \mathfrak{\Omega}
$$

by (49). Hence, modulo $\cong$,

$$
f\left(a_{1}, a_{2}, \cdots, a_{2 n-1}\right)=L_{b_{1}} L_{a_{1}} L_{b_{2}} L_{a_{2}} \cdots L_{a_{2 n-1}} L_{b_{2 n}} L_{a_{2 n}}
$$

is an alternating function of $a_{1}, a_{2}, \cdots, a_{2 n-1} \in \mathfrak{A}_{1}$, and

$$
f\left(a_{1}, \cdots, a_{2 n-1}\right) \in \mathfrak{Q} \quad \text { if } a_{i}=a_{j} \text { for some } i \neq j .
$$

Also $f\left(a_{1}, a_{2}, \cdots, a_{2 n-1}\right)$ is in $\mathfrak{Q}$ if any $a_{i}$ is in $\mathfrak{B}$. If $2 n-1>\operatorname{dim} \mathfrak{A} / \mathfrak{B}$, the $a_{i}$ cannot be independent modulo $F 1+\mathfrak{B}$. Hence $f\left(a_{1}, a_{2}, \cdots, a_{2 n-1}\right)$ is in $\mathfrak{Q}$ when $2 n-1>\operatorname{dim} \mathfrak{A} / \mathfrak{B}$, and (48) implies that

$$
\mathfrak{B}^{\langle n+1\rangle} \subseteq \mathfrak{B} \mathfrak{Q}+\mathfrak{B}^{3}=\mathfrak{B}^{3} \text {. }
$$

This establishes (47), and completes the proof of the theorem.

THEOREM 4 (Wedderburn principal theorem for standard algebras). Let $\mathfrak{A}$ be a finite-dimensional standard algebra over $F$ of characteristic $\neq 2$, and let $\mathfrak{R}$ be the radical of $\mathfrak{A}$. If $\mathfrak{A} / \mathfrak{N}$ is separable, then

$$
\mathfrak{A}=\mathfrak{S}+\mathfrak{N} \quad(\text { direct } \text { sum })
$$

where $\mathfrak{S}$ is a subalgebra of $\mathfrak{A}, \mathfrak{S} \cong \mathfrak{A} / \mathfrak{N}$. 
Proof. We may assume that the solvable ideal $\mathfrak{N}$ is $\neq 0$. If $\mathfrak{R}^{\langle 1\rangle}=\mathfrak{R}$, then $\mathfrak{N}=\mathfrak{N}^{\langle 1\rangle}=\mathfrak{R}^{\langle 2\rangle}=\cdots=\mathfrak{R}^{\langle k\rangle}=0$ for some $k$, since $\mathfrak{R}$ is Penico solvable by Theorem 3. Hence the ideal $\mathfrak{N}^{\langle 1\rangle}$ of $\mathfrak{A}$ is properly contained in $\mathfrak{N}$, and we may make the usual reduction of the proof of the theorem to the case $\mathfrak{R}^{2}=0$ by an inductive argument based on the dimension of $\mathfrak{A}[1, \mathrm{p}$. 47]. We may also make three further reductions which are typical of proofs of the Wedderburn principal theorem for other classes of algebras [18, pp. 64-65; 7, Chapter VII]. We may assume that $\mathfrak{A}$ contains 1 , and that $F$ is algebraically closed. Finally we may assume that $\mathfrak{U} / \mathfrak{N}$ is (central) simple. For, if $\mathfrak{A} / \mathfrak{N}=$ $\mathfrak{B}_{1} \oplus \cdots \oplus \mathfrak{B}_{r}, \mathfrak{B}_{i}$ simple, the identity elements of the $\mathfrak{B}_{i}$ are pairwise orthogonal idempotents in $\mathfrak{U}^{+} / \mathfrak{N}^{+}=(\mathfrak{U} / \mathfrak{N})^{+}$and may be lifted to pairwise orthogonal idempotents $e_{i}$ satisfying $1=e_{1}+\cdots+e_{r}$ in the commutative Jordan algebra $\mathfrak{A}^{+}$. But then the $e_{i}$ are pairwise orthogonal idempotents in $\mathfrak{A}$. We have seen in (23) that, in the corresponding Peirce decomposition (21), the radical of $\mathfrak{A}_{i i}$ is $\mathfrak{N}_{i i}=\mathfrak{N} \cap \mathfrak{A}_{i i}(i=$ $1, \cdots, r)$. This is sufficient by the usual argument to reduce the proof of the theorem to the case where $\mathfrak{A} / \mathfrak{N}$ is (central) simple.

We know from $\S 1$ that the simple standard algebra $\mathfrak{X} / \mathfrak{N}$ is either associative or a commutative Jordan algebra. Let the degree of the central simple algebra $\mathfrak{A} / \mathfrak{R}$ be $t$. Then, by the lifting of idempotents proved above, $1 \in \mathfrak{A}$ may be written as $1=e_{1}+\cdots+e_{t}$ for pairwise orthogonal idempotents $e_{i}$ in $\mathfrak{U}(i=1, \cdots, t)$. If $t=1$, then $\operatorname{dim} \mathfrak{A} / \mathfrak{R}=$ 1 , and $F 1$ is the desired subalgebra of $\mathfrak{A}$. We shall give separate proofs for the cases $t=2$ and $t \geqq 3$.

Assume that $\mathfrak{U} / \mathfrak{N}$ is a (central) simple commutative Jordan algebra of degree 2. Then, since $F$ is algebraically closed, $\mathfrak{A} / \mathfrak{N}$ has a basis $[1],\left[v_{1}\right], \cdots,\left[v_{m}\right], m \geqq 2$, where $[x]$ denotes the residue class $[x]=$ $x+\mathfrak{R}$ of $x \in \mathfrak{A}$, and where

$$
\left[v_{i}\right]^{2}=[1], \quad\left[v_{i}\right]\left[v_{j}\right]=[0] \quad \text { if } i \neq j(i, j=1, \cdots, m) .
$$

Now $\mathfrak{U}^{+}$is a commutative Jordan algebra with radical $\mathfrak{R}^{+}$, and $\mathfrak{A}^{+} / \mathfrak{N}^{+}=(\mathfrak{U} / \mathfrak{R})^{+}=\mathfrak{U} / \mathfrak{R}$. By a special case of the Wedderburn principal theorem for commutative Jordan algebras, there are elements $u_{1}, \cdots, u_{m}$ in $\mathfrak{U}^{+}$such that

$$
u_{i}^{2}=1, \quad u_{i} \cdot u_{j}=0 \quad \text { for } i \neq j(i, j=1, \cdots, m) .
$$

In the Peirce decomposition (17) of $\mathfrak{A}$ relative to the idempotent $e=$ $\frac{1}{2}\left(1+u_{1}\right)$, we have $u_{i} \in \mathfrak{U}_{\frac{1}{2}}(e)=\mathfrak{A}_{10}+\mathfrak{P}_{\frac{1}{2} \frac{1}{2}}+\mathfrak{U}_{01}$ for $i=2, \cdots, m$. Write

$$
u_{i}=r_{i}+s_{i}+t_{i} \quad(i=2, \cdots, m)
$$

where $r_{i} \in \mathfrak{A}_{10}, s_{i} \in \mathfrak{A}_{\frac{1}{2} \frac{1}{2}}, t_{i} \in \mathfrak{A}_{01}$. Since $\mathfrak{A} / \mathfrak{N}$ is a commutative Jordan 
algebra, we have $\mathfrak{A}_{10}+\mathfrak{A}_{01} \subseteq \mathfrak{R}$ by the final remark in $\S 2$. Since $\mathfrak{N}^{2}=0$, (18) implies that

$$
u_{i} u_{j}=s_{i} s_{j} \in \mathfrak{A}_{1}+\mathfrak{A}_{0}
$$

for $i, j=2, \cdots, m$.

Write $s_{1}=u_{1}=2 e-1$. Then

$$
s_{i}^{2}=1, \quad s_{1} s_{j}=s_{j} s_{1}=0 \quad(i=1, \cdots, m ; j=2, \cdots, m)
$$

and

$$
s_{i} s_{j}=c_{1}+c_{0}, \quad c_{1} \in \mathfrak{A}_{1}, c_{0} \in \mathfrak{A}_{0} \quad(i \neq j ; i, j=2, \cdots, m) .
$$

By (53) and (54) we have $0=u_{i} u_{j}+u_{j} u_{i}=s_{i} s_{j}+s_{j} s_{i}$ for $i \neq j(i, j=$ $2, \cdots, m)$, so that $s_{j} s_{i}=-c_{1}-c_{0}$ and $\left[s_{i}, s_{j}\right]=2 c_{1}+2 c_{0}$. Then (3) implies that $\left[s_{i} e, s_{j}\right]=\left[s_{i}, s_{j}\right] e+s_{i}\left[e, s_{j}\right]$, or $c_{1}+c_{0}=2 c_{1}$, so that $c_{1}=$ $c_{0}=0$, and $s_{i} s_{j}=0$ in (56). Then (56) and (55) imply that $1, s_{1}, \cdots, s_{m}$ form a basis for a subalgebra of $\mathfrak{A}$ which is isomorphic to $\mathfrak{A} / \mathfrak{R}$.

Next assume that $\mathfrak{X} / \mathfrak{N}$ is a (central) simple associative algebra of degree 2 over the algebraically closed field $F$. Then $\mathfrak{U} / \mathfrak{A}$ is isomorphic to the algebra of all $2 \times 2$ matrices over $F$, and $(\mathfrak{U} / \mathfrak{R})^{+}$is a central simple commutative Jordan algebra of degree 2. By the Wedderburn principal theorem for commutative Jordan algebras, $\mathfrak{U}^{+}$ contains elements $u_{1}, u_{2}, u_{3}$, satisfying (53). We have seen that $u_{2}$ is in the Peirce space $\mathfrak{V}_{\frac{1}{2}}(e)=\mathfrak{A}_{10}+\mathfrak{U}_{\frac{1}{2} \frac{1}{2}}+\mathfrak{U}_{01}$ relative to the idempotent $e=\frac{1}{2}\left(1+u_{1}\right)$. Write

$$
u_{2}=a_{10}+a_{\frac{1}{2} \frac{1}{2}}+a_{01}, \quad a_{i j} \in \mathfrak{A}_{i j}(e) .
$$

Since $\mathfrak{X} / \mathfrak{N}$ is associative, we have $\mathfrak{U}_{\frac{1}{2} \frac{1}{2}} \subseteq \mathfrak{R}$. Then $\mathfrak{N}^{2}=0$ and (18) imply that $u_{2}^{2}=a_{10} a_{01}+a_{01} a_{10}$ where $a_{10} a_{01} \in \mathfrak{A}_{1}, a_{01} a_{10} \in \mathfrak{H}_{0}$. But $u_{2}^{2}=$ $1=e+(1-e)$, so that $a_{10} a_{01}=e, a_{01} a_{10}=1-e$. Hence $\mathfrak{A}$ contains elements $u_{11}=e, u_{12}=a_{10}, u_{21}=a_{01}, u_{22}=1-e$ which form the basis for a subalgebra $\mathfrak{S}$ with multiplication table $u_{i j} u_{k l}=\delta_{j k} u_{i l}(i, j, k, l=$ $1,2), \subseteq \subseteq \mathfrak{A} / \mathfrak{N}$ as desired.

Finally we assume that $\mathfrak{A} / \mathfrak{N}$ is a (central) simple standard algebra of degree $t \geqq 3$. We know that $1=e_{1}+\cdots+e_{t}$ for pairwise orthogonal idempotents $e_{i}$ in $\mathfrak{A}$. We wish to show that the idempotents $e_{i}$ and $e_{j}(i \neq j)$ are connected $(i, j=1, \cdots, t)$. We know from (23) that the radical of $\mathfrak{B}=\mathfrak{A}_{i i}+\mathfrak{A}_{i j}+\mathfrak{A}_{j j}=\mathfrak{A}_{1}\left(e_{i}+e_{j}\right)$ in $(22)$ is $\mathfrak{B} \cap \mathfrak{R}$. Now $\mathfrak{B} /(\mathfrak{B} \cap \mathfrak{R}) \cong(\mathfrak{B}+\mathfrak{R}) / \mathfrak{R}=(\mathfrak{U} / \mathfrak{N})_{i i}+(\mathfrak{U} / \mathfrak{R})_{i j}+(\mathfrak{U} / \mathfrak{R})_{j j}$ where subscripts indicate Peirce spaces relative to the pairwise orthogonal idempotents $\left[e_{1}\right], \cdots,\left[e_{t}\right]$ in $\mathfrak{A} / \mathfrak{R}$. But in both the associative and commutative Jordan cases, the latter algebra is a central simple algebra of degree 2. We have already seen that such a residue class algebra may be lifted, so we know that $\mathfrak{B}$ contains a subalgebra with identity $e_{i}+e_{j}$ 
which is a central simple standard algebra of degree 2 . In case $\mathfrak{A} / \mathfrak{N}$ is associative, $\mathfrak{B}$ contains a matric basis $u_{i i}=e_{i}, u_{i j}, u_{j i}, u_{j j}=e_{j}$. Then $u_{i j}+u_{j i} \in\left(\mathfrak{A}_{10}\left(e_{i}\right)+\mathfrak{A}_{01}\left(e_{i}\right)\right) \cap\left(\mathfrak{A}_{10}\left(e_{j}\right)+\mathfrak{A}_{01}\left(e_{j}\right)\right)$ is regular in $\mathfrak{B}$ since $\left(u_{i j}+u_{j i}\right)^{2}=e_{i}+e_{j}$. That is, the idempotents $e_{i}, e_{j}$ are connected with indicator $\varphi=0$. By McCrimmon's results [11, p. 191] which we mentioned in $\S 2$, the algebra $\mathfrak{A}$ itself is associative. But then the Wedderburn principal theorem is known to be true. Similarly, if $\mathfrak{A} / \mathfrak{R}$ is a commutative Jordan algebra, $\mathfrak{B}$ contains $u_{1}=e_{i}-e_{j}, u_{2}, \cdots, u_{m}$ $(m \geqq 2)$ satisfying $u_{k}^{2}=e_{i}+e_{j}, u_{k} u_{l}=0(k \neq l ; k, l=1, \cdots, m)$. Then $u_{2} \in \mathfrak{A}_{\frac{1}{2} \frac{1}{2}}\left(e_{i}\right) \cap \mathfrak{U}_{\frac{1}{2} \frac{1}{2}}\left(e_{j}\right)$ is regular in $\mathfrak{B}$. That is, the idempotents $e_{i}, e_{j}$ are connected with indicator $\varphi=1 / 4$, and the algebra $\mathfrak{A}$ itself is a commutative Jordan algebra, in which case the Wedderburn principal theorem is known to hold. This completes the proof of Theorem 4.

5. The Malcev-Harish-Chandra theorem. For the remainder of this paper we assume that the field $F$ has characteristic 0 . Our results are generalizations to standard algebras of known theorems concerning associative and commutative Jordan algebras of characteristic 0 .

If $D$ is a nilpotent derivation of a nonassociative algebra $\mathfrak{A}$ of characteristic 0 , then

$$
\exp D=I+D+\frac{D^{2}}{2 !}+\frac{D^{3}}{3 !}+\cdots
$$

is an automorphism of $\mathfrak{A}$. Two subalgebras of $\mathfrak{A}$ are called strictly conjugate if one is mapped onto the other by an automorphism of the form $G_{1} G_{2} \cdots G_{k}, G_{i}=\exp D_{i}, D_{i}$ a nilpotent derivation.

THEOREM 5. (Malcev-Harish-Chandra theorem for standard algebras). Let $\mathfrak{A}$ be a finite-dimensional standard algebra of characteristic 0 with Wedderburn decomposition $\mathfrak{A}=\mathfrak{S}+\mathfrak{N}$ as in (52), and let $\mathfrak{M}$ be a semisimple subalgebra of $\mathfrak{A}$. Then $\mathfrak{M}$ is strictly conjugate to a subalgebra of $\mathfrak{\subseteq}$.

Proof. Jacobson has proved this for commutative Jordan algebras $\mathfrak{\Im}[6$, Th. 9.3], and has remarked that the Campbell-Hausdorff formula

$$
\exp D_{1} \exp D_{2}=\exp \left(D_{1}+D_{2}+\frac{1}{2}\left[D_{1}, D_{2}\right]+\cdots\right)
$$

permits one to give the conjugacy by an automorphism $G=\exp D$, $D$ in the radical of the multiplication algebra $\mathfrak{M}(\Im)$. We need to observe that, since the derivations $D_{i}$ which he uses in his proof are 
inner derivations of $\Im$, the Campbell-Hausdorff formula (57) gives conjugacy by $G=\exp D$ where $D$ is an inner derivation of $\Im$.

Now $\mathfrak{M}^{+}$is a semisimple subalgebra of the commutative Jordan algebra $\mathfrak{A}^{+}=\mathfrak{S}^{+}+\mathfrak{R}^{+}$. Then $\mathfrak{M}^{+}$is strictly conjugate to a subalgebra of $\mathfrak{S}^{+}$, the conjugacy being given by $G=\exp D$, where $D$ is a nilpotent inner derivation of $\mathfrak{A}$. By the Corollary to Theorem $1, D$ is actually a derivation of $\mathfrak{A}$. Hence $G$ is an automorphism of $\mathfrak{A}$, and $G$ maps $\mathfrak{M}$ onto a subalgebra of $\mathfrak{S}$.

COROLLARY. If a standard algebra $\mathfrak{A}$ of characteristic 0 has Wedderburn decompositions $\mathfrak{A}=\mathfrak{S}+\mathfrak{R}=\mathfrak{S}_{1}+\mathfrak{R}$, then $\mathfrak{S}_{1}$ is strictly conjugate to $\subseteq$.

THEOREM 6. If $\mathfrak{A}$ is a semisimple subalgebra of a finite-dimensional standard algebra $\mathfrak{B}$ of characteristic 0 , then any derivation of $\mathfrak{A}$ into $\mathfrak{B}$ can be extended to an inner derivation

$$
D=R_{d}-L_{d}+\sum_{i}\left[L_{x_{i}}, R_{z_{i}}\right], \quad d, x_{i}, z_{i} \in \mathfrak{B},
$$

of $\mathfrak{B}$.

Proof. This result is known for commutative Jordan algebras [6, Th. 9.1]. Now $\mathfrak{A}^{+}$is a semisimple subalgebra of the commutative Jordan algebra $\mathfrak{B}^{+}$. Any derivation of $\mathfrak{X}$ into $\mathfrak{B}$ is also a derivation of $\mathfrak{A}^{+}$into $\mathfrak{B}^{+}$, and can therefore be extended to an inner derivation $D$ of $\mathfrak{B}^{+}$. By the Corollary to Theorem $1, D$ is a derivation of $\mathfrak{A}$. Since $D$ is a sum of derivations (30), $D$ has the form (58) and is inner by Theorem 2 .

Theorem 6 is equivalent to the first Whitehead lemma for standard algebras of characteristic 0 , which involves the notion of standard bimodule $\mathfrak{B}$ or representation $(S, T)$ of a standard algebra. We omit the definitions which are determined easily from the general definitions in [18, pp. 25-26]. The first Whitehead lemma may then be stated as follows: Let $\mathfrak{A}$ be a semisimple standard algebra of characteristic 0 with representation $(S, T)$ acting in $\mathfrak{B}$. Let $\nu$ be a derivation of $\mathfrak{A}$ into $\mathfrak{B}$ (a "one-cocycle"): $\nu$ is a linear mapping of $\mathfrak{U}$ into $\mathfrak{B}$ satisfying

$$
\nu(x y)=x \nu(y)+\nu(x) y=\nu(y) T_{x}+\nu(x) S_{y}
$$

for all $x, y$ in $\mathfrak{U}$. Then, if $\mathfrak{B}$ is the semidirect sum $\mathfrak{B}=\mathfrak{U}+\mathfrak{B}$, there exist $x_{i} \in \mathfrak{A}$ and $d, z_{i} \in \mathfrak{B}$ such that

$$
\nu(y)=y d-d y+\sum_{i}\left(x_{i}, y, z_{i}\right) ;
$$

that is, $\nu(y)=y D$ where $D$ is the inner derivation (58) of $\mathfrak{B}$. 
The restriction to characteristic 0 is not necessary in Theorem 6 and the first Whitehead lemma. With a more complicated hypothesis, more general results may be obtained by using [4, Th. 2] instead of [6] in the proof.

We conclude with a generalization of the theorem in [14].

LEMma 2. The radical $\mathfrak{R}$ of any finite-dimensional standard algebra $\mathfrak{A}$ of characteristic 0 is characteristic; that is,

$$
\mathfrak{R D} \cong \mathfrak{R}
$$

for every derivation $D$ of $\mathfrak{A}$.

Proof. Albert has shown that, for characteristic 0,

$$
\mathfrak{N}=\left\{x \mid \text { trace } R_{x y}=0 \text { for all } y \in \mathfrak{N}\right\}
$$

[2, p. 581]. Then $D \in \mathfrak{D}(\mathfrak{U}), x \in \mathfrak{R}$ imply trace $R_{(x D) y}=$ trace $R_{(x y) D-x(y D)}=$ trace $\left[R_{x y}, D\right]$ - trace $R_{x(y D)}=0$ for all $y$ in $\mathfrak{A}$ by (24), (25) and (60). Hence $x D \in \mathfrak{R}$, implying (59).

Lemma 3. Let $\subseteq$ be a finite-dimensional semisimple standard algebra of characteristic 0,3 be the center of $\mathfrak{S}, \mathfrak{P}$ the associator subspace of $\mathfrak{S}$ (spanned by all associators in $\mathfrak{S}$ ), and $\mathfrak{S}^{\prime}=[\mathfrak{S}, \mathfrak{S}]$ the commutator subspace of $\mathfrak{\subseteq}$. Then $\subseteq$ is the direct sum

$$
\mathfrak{S}=3+\mathfrak{S}^{\prime}+\mathfrak{P} \text {. }
$$

Proof. Since it is sufficient to show this for each simple component, we may assume that $\subseteq$ is simple. If $\subseteq$ is associative, we have $\mathfrak{P}=0$, and it is well known that $\subseteq$ is the direct sum $\subseteq=3+\mathfrak{S}^{\prime}$. If $\subseteq$ is a commutative Jordan algebra, we have $\mathfrak{S}^{\prime}=0$, and $\subseteq=3+\mathfrak{P}$ [14, p. 292].

THeOREm 7. Let $\mathfrak{A}$ be a finite-dimensional standard algebra of characteristic 0 , and $\mathfrak{D}(\mathfrak{H})$ be its derivation algebra. Then $\mathfrak{H}$ is semisimple with each simple component of dimension $\neq 3$ over its center if and only if $\mathfrak{D}(\mathfrak{U})$ is semisimple or 0.

Proof. If $\mathfrak{U}=\mathfrak{S}_{1} \oplus \cdots \oplus \mathfrak{S}_{r}$ is semisimple, each simple component $\mathfrak{S}_{i}$ is either associative or a commutative Jordan algebra, and $\mathfrak{D}(\mathfrak{A})$ is the direct sum of the $\mathfrak{D}\left(\mathfrak{S}_{i}\right)$. The latter are all known to be semisimple or 0 , except when $\mathfrak{S}_{i}$ is (a commutative Jordan algebra) of dimension 3 over its center, in which case $\mathfrak{D}\left(\mathfrak{S}_{i}\right) \neq 0$ is abelian. Therefore, in order to prove the theorem, it is sufficient to show that, if $\mathfrak{D}(\mathfrak{A})$ is semisimple or 0 , then the radical $\mathfrak{N}$ of $\mathfrak{U}$ is 0 . 
Let $\mathfrak{D}_{1}$ be the subspace of $\mathfrak{D}(\mathfrak{U})$ spanned by the derivations (30), $x, y$ in $\mathfrak{R}$. Then $\mathfrak{D}_{1}$ is a solvable ideal of $\mathfrak{D}\left(\mathfrak{A}^{+}\right)$[14, p. 292]. But $\mathfrak{D}_{1} \subseteq \mathfrak{D}(\mathfrak{U}) \subseteq \mathfrak{D}\left(\mathfrak{U}^{+}\right)$, so $\mathfrak{D}_{1}$ is a solvable ideal of $\mathfrak{D}(\mathfrak{A})$. Hence $\mathfrak{D}_{1}=0$. Let $\mathfrak{D}_{2}$ be the subspace of $\mathfrak{D}(\mathfrak{U})$ spanned by the derivations $(30), x$ in $\mathfrak{A}, y$ in $\mathfrak{R}$. Then $\mathfrak{D}_{2}$ is also a solvable ideal of $\mathfrak{D}\left(\mathfrak{A}^{+}\right)[14$, p. 293], and similarly $\mathfrak{D}_{2}=0$. That is,

$$
R_{[x, z]}-L_{[x, z]}+4\left[L_{x}, R_{z}\right]=0 \quad \text { for all } x \in \mathfrak{A}, z \in \mathfrak{R} .
$$

Let

$$
\mathfrak{D}_{3}=\left\{R_{z}-L_{z} \mid z \in \mathfrak{N}\right\} \text {. }
$$

Lemma 2 implies that $\mathfrak{D}_{3}$ is an ideal of $\mathfrak{D}(\mathfrak{U}):\left[R_{z}-L_{z}, D\right]=R_{z D}-$ $L_{z D} \in \mathfrak{D}_{3}$ for all $z \in \mathfrak{R}, D \in \mathfrak{D}(\mathfrak{A})$ by (25), (26) and (63). Also $\mathfrak{D}_{3}$ is solvable. For it is easy to see by induction on $k$ that the $k$ th derived algebra $\mathfrak{D}_{3}^{(k)}$ of $\mathfrak{D}_{3}$ is spanned by derivations of the form (28) where $d$ is a product of $2^{k}$ elements of $\mathfrak{R}$. Since $\mathfrak{R}$ is nilpotent, $\mathfrak{D}_{3}^{(k)}=0$ for some $k$. Hence $\mathfrak{D}_{3}=0$, implying

$$
R_{z}=L_{z}
$$

for all $z \in \mathfrak{N}$.

Then (62) and (64) imply that

$$
\left[L_{x}, R_{z}\right]=0 \quad \text { for all } x \in \mathfrak{A}, z \in \mathfrak{R} .
$$

It follows from (64) and (65) that $\mathfrak{R}$ is contained in the center $\mathfrak{S}$ of $\mathfrak{A}$. Let $\mathfrak{A}=\mathfrak{S}+\mathfrak{N}$ be a Wedderburn decomposition (52) of $\mathfrak{A}$. Then (5) is the direct sum

$$
\mathfrak{c}=3+\mathfrak{R}
$$

where 3 is the center of $\mathfrak{S}$. It follows from (61) and (66) that

$$
\mathfrak{U}=\mathfrak{S}^{\prime}+\mathfrak{P}+\mathfrak{S} \text {. }
$$

Now $z \in \mathfrak{N} \subseteq \mathfrak{C}$ implies $z\left[a_{1}, a_{2}\right]=\left[z a_{1}, a_{2}\right]=0$ and $z\left(a_{1}, a_{2}, a_{3}\right)=\left(z a_{1}, a_{2}, a_{3}\right)=$ 0 for all $a_{i} \in \mathfrak{A}$, since $z a_{1} \in \mathfrak{N}$. Hence $\mathfrak{N} \mathfrak{S}^{\prime}=0, \mathfrak{R} \mathfrak{P}=0$, and $\mathfrak{S}^{\prime} \subseteq \mathfrak{S}^{\prime}$,

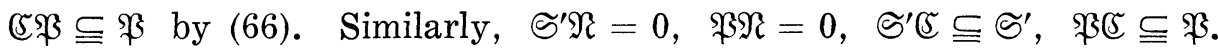
Let $D_{\mathbb{S}}$ be any derivation of the commutative associative algebra $\sqrt{5}$ (into itself). Let $D$ be the linear extension of $D_{\mathbb{5}}$ to $\mathfrak{A}$ defined by $\mathfrak{S}^{\prime} D=\mathfrak{P} D=0$. Then $D$ is a derivation of $\mathfrak{A}$, as may be checked by the same type of computation as given in [14, p. 294]. That is, every derivation of $\mathfrak{C}$ is induced by a derivation of $\mathfrak{A}$, and the proof of the theorem is completed as in [14, p. 294]: $\mathscr{D}(\mathbb{S})$ is a homomorphic image of $\mathfrak{D}(\mathfrak{U})$, so is either semisimple or 0 . Then Hochschild's result for associative algebras [5, Th. 4.5] implies that $\sqrt{5}$ is semisimple, so its radical $\mathfrak{N}=0$. This completes the proof of Theorem 7 . 
The Wedderburn principal theorem does not hold for noncommutative Jordan algebras (even for characteristic 0 , or for $\mathfrak{N}^{2}=0$ ), as may be seen by a 5-dimensional example [18, p. 147; 16, p. 477]. However, all of the theorems of this paper have valid analogues for alternative algebras [18, 15]. It would be interesting to know whether there is a class of nonassociative algebras, containing all alternative algebras as well as all commutative Jordan algebras, for which the analogous theorems are true. By virtue of the example cited above, such a class cannot be as inclusive as the class of all noncommutative Jordan algebras. It has been suggested that strongly homogeneous algebras [3, p. 109; 9, p. 356] might constitute such a class. However, the same example disproves this conjecture. For this particular 5-dimensional algebra is a quadratic algebra in which it is easy to compute that $(x y)^{-1}=y^{-1} x^{-1}$ for generically independent elements $x, y$. Hence the algebra is strongly homogeneous by Theorem 10 of [9]. See [3, p. 131] for a related conjecture, which is not so easy to settle since it requires the existence of an unspecified set of identities.

\section{REFERENCES}

1. A. A. Albert, Structure of algebras, Amer. Math. Soc. Colloq. Publ., Vol. 24, New York, 1939.

2. - Power-associative rings, Trans. Amer. Math. Soc. 64 (1948), 552-593.

3. H. Braun and M. Koecher, Jordan-algebren, Springer-Verlag, 1966.

4. Bruno Harris, Derivations of Jordan algebras, Pacific J. Math. 9 (1959), 495-512.

5. G. P. Hochschild, Semisimple algebras and generalized derivations, Amer. J. Math. 64 (1942), 677-694.

6. N. Jacobson, General representation theory of Jordan algebras, Trans. Amer. Math. Soc. 70 (1951), 509-548.

7. - Structure and Representations of Jordan algebras, Amer. Math. Soc. Colloq. Publ. (to appear)

8. Erwin Kleinfeld, Standard and accessible rings, Canad. J. Math. 8 (1956), 335-340.

9. M. Koecher, On homogeneous algebras, Bull. Amer. Math. Soc. 72 (1966), 347-357.

10. K. McCrimmon, Norms and noncommutative Jordan algebras, Pacific J. Math. 15 (1965), 925-956.

11. - Structure and representations of noncommutative Jordan algebras, Trans. Amer. Math. Soc. 121 (1966), 187-199.

12. A. J. Penico, The Wedderburn principal theorem for Jordan algebras, Trans. Amer. Math. Soc. 70 (1951), 404-420.

13. - On the structure of standard algebras (abstract), Proc. International Congress of Mathematicians, Cambridge, Mass., 1950, vol. 1, p. 320; Amer. Math. Soc., Providence, R.I., 1952.

14. R. D. Schafer, A theorem on the derivations of Jordan algebras, Proc. Amer. Math. Soc. 2 (1951), 290-294.

15. - Representations of alternative algebras, Trans. Amer. Math. Soc. 72 (1952), 1-17.

16. Structure and representation of nonassociative algebras, Bull. Amer. Math. Soc. 61 (1955), 469-484.

17. - On noncommutative Jordan algebras, Proc. Amer. Math. Soc. 9 (1958), $110-117$. 
18. — An Introduction to nonassociative algebras, Academic Press, New York and London, 1966.

Received October 24, 1967. This research was supported by National Science Foundation grant GP-6982.

Massachusetts Institute of TeChNology

Cambridge, Massachusetts 




\section{Pacific Journal of Mathematics \\ Vol. 29, No. 1 \\ May, 1969}

Jorge Alvarez de Araya, A Radon-Nikodým theorem for vector and operator

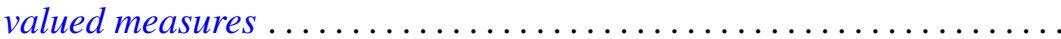

Deane Eugene Arganbright, The power-commutator structure of finite

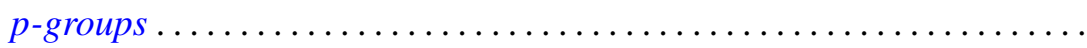

Richard Eugene Barlow, Albert W. Marshall and Frank Proschan, Some

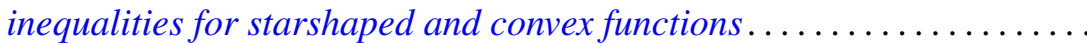

David Clarence Barnes, Some isoperimetric inequalities for the eigenvalues

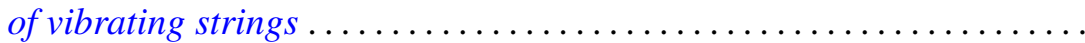

David Hilding Carlson, Critical points on rim-compact spaces ...........

Allan Matlock Weber Carstens, The lattice of pretopologies on an arbitrary

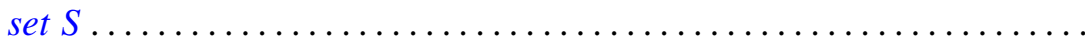

S. K. Chatterjea, A bilateral generating function for the ultraspherical

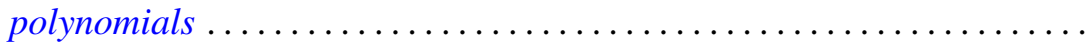

Ronald J. Ensey, Primary Abelian groups modulo finite groups ......... 77

Harley M. Flanders, Relations on minimal hypersurfaces ............ 83

Allen Roy Freedman, On asymptotic density in n-dimensions........... 95

Kent Ralph Fuller, On indecomposable injectives over artinian rings...... 115

George Isaac Glauberman, Normalizers of p-subgroups in finite groups . . . 137

William James Heinzer, On Krull overrings of an affine ring ........... 145

John McCormick Irwin and Takashi Ito, A quasi-decomposable abelian group without proper isomorphic quotient groups and proper isomorphic subgroups.

Allan Morton Krall, Boundary value problems with interior point boundary conditions

John S. Lowndes, Triple series equations involving Laguerre

polynomials

Philip Olin, Indefinability in the arithmetic isolic integers

Ki-Choul Oum, Bounds for the number of deficient values of entire functions whose zeros have angular densities..

R. D. Schafer, Standard algebras ....................

Wolfgang M. Schmidt, Irregularities of distribution. III.

Richard Alfred Tapia, An application of a Newton-like method to the Euler-Lagrange equation 